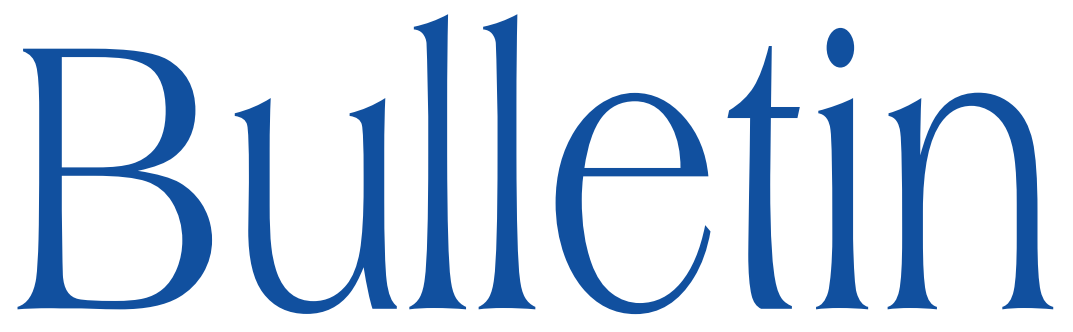

de la SOCIÉTÉ MATHÉMATIQUE DE FRANCE

\title{
UNE NOUVELLE PREUVE DE LA CONCORDANCE DES CLASSES DÉFINIES PAR M.-H. SCHWARTZ ET PAR R. MACPHERSON
}

Paolo Aluffi \& Jean-Paul Brasselet

\section{Tome 136}

Fascicule 2 


\title{
UNE NOUVELLE PREUVE DE LA CONCORDANCE DES CLASSES DÉFINIES PAR M.-H. SCHWARTZ ET PAR R. MACPHERSON
}

\author{
Par Paolo Aluffi \& Jean-Paul Brasselet
}

À Marie-Hélène Schwartz

RÉsumé. - Nous donnons une courte démonstration de ce que les classes des variétés singulières définies par Marie-Hélène Schwartz au moyen des «champs radiaux » coïncident avec la notion fonctorielle définie par Robert MacPherson.

\begin{abstract}
A$ new proof of the agreement between the classes defined by M.H. Schwartz and R. MacPherson)

We give a short proof of the fact that the Chern classes for singular varieties defined by Marie-Hélène Schwartz by means of 'radial frames' agree with the functorial notion defined by Robert MacPherson.
\end{abstract}

\section{Introduction}

Marie-Hélène Schwartz ([6], [7]) a défini, au milieu des années 60, une notion de classes de Chern pour les variétés singulières en cohomologie relative, ou, via dualité d'Alexander, en homologie à coefficients entiers. La méthode

Texte reçu le 6 juillet 2006, révisé le 9 janvier 2007

Paolo Aluffi, Mathematics Department, Florida State University, Tallahassee FL 32306, U.S.A. - E-mail : aluffi@math.fsu.edu

Jean-Paul Brasselet, IML - CNRS, Case 907, Luminy, 13288 Marseille Cedex 9, France - $\quad$ E-mail : jpb@iml.univ-mrs.fr

Classification mathématique par sujets (2000). — 14C17, 57R20.

Mots clefs. - Classes de Chern, variétés singulières, conjecture de Grothendieck-Deligne, champs radiaux. 
utilisée est la théorie d'obstruction appliquée à des champs de repères dont le comportement est contrôlé le long de la partie singulière.

Par la suite, le travail d'Alexander Grothendieck en vue de montrer un 'théorème de Riemann-Roch discret' a été à l'origine de la conjecture de l'existence (en caractéristique zéro) d'une théorie fonctorielle de classes de Chern, comme transformation naturelle du foncteur des fonctions constructibles dans une théorie d'homologie convenable. Cette conjecture est connue sous le nom de conjecture de Deligne-Grothendieck. Celle-ci a été résolue, dans les années 70, par Robert MacPherson [5], lequel a ainsi donné une autre construction de classes de Chern pour les variétés singulières.

Le travail de R. MacPherson est indépendant de celui de M.H. Schwartz, bien que les deux notions aient des points communs : d'une part, les deux notions de classes spécialisent aux classes de Chern usuelles pour les variétés lisses ; d'autre part, le fait que le résultat de M.H. Schwartz étende le Théorème de Poincaré-Hopf aux variétés singulières peut être considéré comme un aspect de la propriété de fonctorialité satisfaite par les classes de MacPherson.

Il était donc naturel de conjecturer que ces deux notions coïncident, ce qui a été démontré en 1979 par M.H. Schwartz et le second auteur du présent article :

ThÉORÈme 1.1 ([2]). - Les classes de Chern homologiques de Schwartz et de MacPherson sont égales.

La méthode de démonstration consiste à relier les indices des champs radiaux et les classes de Schwartz aux ingrédients principaux de la construction des classes de MacPherson, à savoir l'obstruction d'Euler locale et la classe de Chern-Mather.

Dans cet article, nous montrons plus directement l'égalité des classes de Schwartz et de MacPherson, sans nous servir des autres invariants des singularités. Ce nouveau point de vue est inspiré par une nouvelle expression de la notion de fonctorialité des classes de Chern en termes de classes définies pour les variétés lisses (mais non nécessairement complètes), obtenue par le premier auteur [1]. En fait, des développements ultérieurs ont rendu l'argument indépendant de cette référence, ce qui permet de présenter la présente version de façon autonome.

Dans la section 2, on montre qu'une classe définie pour les variétés $X$ (éventuellement singulières) coïncide nécessairement avec la notion fonctorielle si

- elle peut être écrite comme somme des contributions des strates d'une stratification de $X$;

- la contribution d'une strate non singulière $S$ est préservée par morphismes respectant les stratifications; et

- la classe coïncide avec la classe de Chern du fibré tangent d'une variété complète non singulière.

TOME $136-2008-\mathrm{N}^{\mathrm{O}} 2$ 
Dans la section 3 on remarque que les classes définies par Marie-Hélène Schwartz satisfont ces conditions, et le fait qu'elles coïncident avec les classes de MacPherson en résulte immédiatement.

\section{Une caractérisation des classes de Chern fonctorielles}

Dans cette section, nous travaillons sur un corps arbitraire algébriquement clos de caractéristique zéro, et dans le groupe de Chow $A_{*}$. Les résultats sont valables a fortiori pour les variétés complexes, en homologie.

2.1. - Soit $\tilde{c}(X) \in A_{*} X$ une classe définie pour toutes les variétés (éventuellement singulières) $X$, comme une somme de contributions d'une décomposition de $X$ en union finie disjointe de variétés (éventuellement incomplètes) non singulières $S_{i}$ :

$$
X=\coprod_{i} S_{i}: \tilde{c}(X)=\sum_{i} \tilde{c}\left(S_{i}, X\right)
$$

on dit que de telles décompositions sont admissibles (pour $\tilde{c}$ ). Comme nous le verrons, dans certains cas toute décomposition de $X$ comme union finie disjointe de sous-variétés non singulières peut être admissible. Dans d'autres situations, des restrictions peuvent être imposées sur les décompositions pour être admissibles : par exemple, on peut demander aux variétés $S_{i}$ d'être éléments d'une stratification de Whitney de $X$.

Nous supposons que les strates d'un diviseur à croisements normaux forment une décomposition admissible. Plus précisément : si $D$ est un diviseur à croisements normaux simples, de composantes non singulières $D_{j}, j \in J$, d'une variété non singulière $Y$, on suppose que

$$
\coprod_{I \subset J} D_{I}^{\circ}
$$

est une décomposition admissible de $Y$, où $D_{I}^{\circ}$ désigne

$$
\left(\cap_{j \in I} D_{j}\right) \backslash\left(\cup_{j \notin I} D_{j}\right) .
$$

(Par exemple, $D_{\varnothing}^{\circ}$ est le complémentaire de $D$ dans $Y$ ).

Cette propriété sera immédiatement satisfaite pour les décompositions que nous considérerons.

DÉfinition 2.1. - On dit que la donnée, pour toute variété algébrique $X$, d'une classe $\tilde{c}(X) \in A_{*} X$ comme ci-dessus est localement déterminée si la condition suivante est réalisée : 
- Si $f: Y \rightarrow X$ est un morphisme propre, $S$ et $T:=f^{-1}(S)$ resp. sont éléments d'une décomposition admissible de $X$, resp. $Y$, et $f$ se restreint à un isomorphisme $T \rightarrow S$, alors

$$
f_{*} \tilde{c}(T, Y)=\tilde{c}(S, X)
$$

EXEMPLE 2.2 (La classe fonctorielle). - On désigne par $c_{*}(X) \in A_{*}(X)$ la classe définie par MacPherson dans [5] (voir [3], §19.1.7, pour l'adaptation de la définition au groupe de Chow $A_{*}(X)$, et [4] pour l'extension aux corps arbitraires algébriquement clos de caractéristique zéro). Rappelons que cette classe est la valeur

$$
c_{*}(X):=c_{*}\left(\mathbb{1}_{X}\right) \in A_{*} X
$$

prise, sur la fonction caractéristique constante $\mathbb{1}_{X}$, par une transformation naturelle

$$
c_{*}: F \rightarrow A_{*}
$$

$\mathrm{du}$ foncteur des fonctions constructibles dans le foncteur groupe de Chow. Ici $F(X)$ est le groupe des fonctions constructibles à valeurs entières sur $X$; si $g: Y \rightarrow X$ est une application propre, l'image directe $g_{*}(\varphi)$ de la fonction constructible $\varphi=\sum_{Z} m_{Z} \mathbb{1}_{Z} \in F(Y)$ est définie comme la fonction sur $X$ dont la valeur en $p \in X$ est

$$
g_{*}(\varphi)(p):=\sum m_{Z} \chi\left(g^{-1}(p) \cap Z\right) .
$$

Ici $\chi$ désigne la caractéristique d'Euler-Poincaré topologique, sur $\mathbb{C}$; pour l'extension aux autres corps de caractéristique zéro voir [4] ou [1].

Si $S$ désigne une sous-variété quelconque (en particulier non singulière) de $X$, on pose

$$
c_{*}(S, X):=c_{*}\left(\mathbb{1}_{S}\right) \in A_{*} X .
$$

Alors toute décomposition de $X$ comme union finie disjointe de sous-variétés non singulières est admissible pour $c_{*}$. En effet, si $X=\coprod_{i} S_{i}$ alors $\mathbb{1}_{X}=\sum_{i} \mathbb{1}_{S_{i}}$ et donc

$$
c_{*}(X)=c_{*}\left(\mathbb{1}_{X}\right)=\sum_{i} c_{*}\left(\mathbb{1}_{S_{i}}\right)=\sum_{i} c_{*}\left(S_{i}, X\right) .
$$

De plus, $c_{*}$ est localement déterminée. En effet, soit $f: Y \rightarrow X$ une application propre qui se restreint à un isomorphisme $T \rightarrow S$. Alors

$$
f_{*} c_{*}(T, Y)=f_{*} c_{*}\left(\mathbb{1}_{T}\right)=c_{*} f_{*}\left(\mathbb{1}_{T}\right)=c_{*}\left(\mathbb{1}_{S}\right)=c_{*}(S, X)
$$

puisque $c_{*}$ est une transformation naturelle, et par définition de l'image directe des fonctions constructibles. 
ThÉORÈme 2.3. - Supposons que c̃ soit localement déterminée, et que

$$
\tilde{c}(V)=c(T V) \cap[V]
$$

pour toute variété non-singulière complète $V$. Alors $\tilde{c}$ coïncide avec la classe fonctorielle $c_{*}$.

Preuve. Soit $X=\coprod_{i} S_{i}$ une décomposition admissible de $X$ pour $\tilde{c}$; il suffit de montrer que $\tilde{c}\left(S_{i}, X\right)=c_{*}\left(S_{i}, X\right)$.

Si $S$ est un élément de la décomposition, notons $\bar{S}$ son adhérence dans $X$, et considérons $f: Y \rightarrow \bar{S}$ une résolution de $\bar{S}$, telle que le complément de $T:=f^{-1}(S)$ dans $Y=\bar{T}$ soit un diviseur $D$ à croisements normaux, de composantes non singulières $D_{j}, j \in J$. Comme $\tilde{c}$ et $c_{*}$ sont toutes deux localement déterminées, il suffit de montrer que

$$
\tilde{c}(T, Y)=c_{*}(T, Y) \in A_{*} Y .
$$

On a

$$
Y=\coprod_{I \subset J} D_{I}^{\circ}
$$

d'où

$$
\tilde{c}(Y)=\sum_{I \subset J} \tilde{c}\left(D_{I}^{\circ}, Y\right)
$$

ce qui, par le principe d'« inclusion-exclusion », donne

$$
\tilde{c}(T, Y)=\tilde{c}\left(D_{\varnothing}^{\circ}, Y\right)=\sum_{I \subset J}(-1)^{|I|} \tilde{c}\left(D_{I}, Y\right)
$$

où $D_{I}=\cap_{j \in I} D_{j}$, et nous notons par $\tilde{c}\left(D_{I}, Y\right)$ la somme $\sum_{K \supset I} \tilde{c}\left(D_{K}^{\circ}, Y\right)$. Chaque $D_{I}$ est complète et non singulière (puisque $D$ est un diviseur à croisements normaux), donc l'inclusion $\iota: D_{I} \rightarrow Y$ est propre. Pour $K \supset I$ on a

$$
\tilde{c}\left(D_{K}^{\circ}, Y\right)=\iota_{*} \tilde{c}\left(D_{K}^{\circ}, D_{I}\right)
$$

puisque $\tilde{c}$ est localement déterminée, et donc

$$
\tilde{c}\left(D_{I}, Y\right)=\sum_{K \supset I} \iota_{*} \tilde{c}\left(D_{K}^{\circ}, D_{I}\right)=\iota_{*} \tilde{c}\left(D_{I}\right)=\iota_{*}\left(c\left(T D_{I}\right) \cap\left[D_{I}\right]\right) .
$$

En utilisant les mêmes arguments, on a la même expression pour $c_{*}(T, Y)$, d'où le résultat.

\section{Les classes de Schwartz}

Nous supposons maintenant que le corps de base est $\mathbb{C}$, et nous travaillons en homologie à coefficients entiers ; les considérations de la section précédante s'appliquent dans ce contexte, via l'application canonique du groupe de Chow dans l'homologie. 
3.1. - Notons $\tilde{c}$ la classe définie par Marie-Hélène Schwartz dans [6], [7]. Cette classe coïncide avec la classe de Chern (homologique) du fibré tangent pour les variétés non singulières complètes, et se calcule, dans le cas général, comme somme des contributions des strates $S_{i}$ d'une stratification de Whitney de $X$ :

$$
\tilde{c}(X):=\sum_{i} \tilde{c}\left(S_{i}, X\right) .
$$

Autrement dit, les stratifications de Whitney sont des décompositions admissibles pour $\tilde{c}$.

Rappelons brièvement la définition des classes de Schwartz. La variété $X$ est plongée dans une variété non singulière $M$, stratifiée par $M \backslash X$ et par les strates $S_{i}$ de $X$. Pour $x \in M$, on note $E(x)$ le sous-espace de l'espace tangent $T_{x} M$ formé des vecteurs tangents à la strate de $M$ contenant $x$. La collection des sous-espaces $E(x)$ détermine un sous-espace $E$ du fibré tangent $T M$. Ce n'est plus un fibré mais la notion d'une section de $E$ est bien définie, comme section de $T M$ à valeurs dans $E$. On considère alors l'espace $E_{r}$ des $r$-repères ordonnés de vecteurs tangents aux strates de $M$. C'est un sous-espace du fibré $T_{r} M$ des $r$-repères ordonnés de $T M$.

Considèrons aussi une triangulation $K$ de $M$ compatible avec la stratification, et une décomposition cellulaire $D$ de $M$, duale de $K$. Les cellules de $D$ sont transverses aux strates.

En dimension $r-1$, la classe de Schwartz est définie comme l'obstruction à la construction d'une section particulière $Z_{r}$ de $E_{r}$ sur $X$, appelée champ de $r$-repères radial. C'est en particulier une section de $T_{r} M$ sans singularité sur le squelette de dimension (réelle) $2 m-2 r+1$ de la décomposition cellulaire duale $D$ et avec singularités isolées sur le $D$-squelette de dimension $2(m-r+1)$. La construction est faite par récurrence sur la dimension des strates :

Les strates de dimension complexe (strictement) inférieure à $r-1$ n'apparaissent pas. Considérons une strate de dimension $(r-1)$. Les cellules de dimension (réelle) $2(m-r+1)$ intersectent une telle strate en un point (lorsque l'intersection est non vide). Sur une telle cellule, le $r$-repère est construit comme champ radial, et donc d'indice +1 . Puisque $\operatorname{dim}_{\mathbb{C}} S=r-1$, on a alors :

$$
\tilde{c}(S, X)=\sum_{K_{\alpha} \subset S} K_{\alpha}^{r-1}
$$

Supposons que la construction soit déjà effectuée sur les strates de dimension inférieure à celle de $S$. Le $r$-repère $Z_{r}$ est défini sur les strates du bord de $S$, avec des singularités $a_{j}$. Notons $U$ le voisinage «cellulaire» de $\partial S$, composé des cellules de $D$ duales des simplexes de $\partial S$. La construction s'effectue de façon à ce que, dans $U$, l'extension de $Z_{r}$, encore notée $Z_{r}$ n'a pas d'autre singularité que les points $a_{j}$. D'après la construction des champs radiaux et comme prouvé dans Schwartz [8], le $r$-repère $Z_{r}$ satisfait aux propriétés suivantes :

TOME $136-2008-\mathrm{N}^{\mathrm{O}} 2$ 
1) L'indice $I\left(Z_{r}, a_{j}\right)$ est le même, calculé comme section du fibré tangent à la strate de $a_{j}$ ou comme section de $T M$,

2) Le repère $Z_{r}$ est sortant du voisinage cellulaire $U$. Cela signifie que sur $\partial U \cap S$, le $r$-repère $Z_{r}$ est tangent à $S$ et entrant dans $S \backslash U$,

3) Deux champs radiaux sont homotopes sur $\partial U \cap S$, comme sections de $T_{r} S$.

En particulier, ce dernier point est démontré localement dans [8], §6.3 (Théorème 6.3.2, Homotopie entre deux champs radiaux au voisinage d'une strate) et globalement dans [8], Chapitre 9 (Théorème 9.1, Théorème global d'homotopie).

On étend alors $Z_{r}$, défini dans $S \cap U$, dans l'intérieur de $S$, avec singularités isolées $a$ situées dans l'intersection de $S$ avec les $D$-cellules de dimension $2(m-$ $r+1)$. Autrement dit, les points $a$ sont situés dans l'intersection de $S$ avec les $D$-cellules duales des $K$-simplexes de dimension $2(r-1)$ contenus dans $S \backslash U$.

La contribution $\tilde{c}(S, X)$ de la strate $S$ se calcule en termes des indices $I\left(Z_{r}, a\right)$ en ces points singuliers $a$ de $Z_{r}$, situés dans $S \backslash \partial S$.

$$
\tilde{c}(S, X):=\sum_{K_{\alpha} \subset S \backslash \partial S} \mu_{\alpha} K_{\alpha}^{r-1}
$$

où les simplexes $K_{\alpha}$ sont de dimension $2(r-1)$ et

$$
\mu_{\alpha}=\sum_{a \in D_{\alpha} \cap S} I\left(Z_{r}, a\right)
$$

où la cellule $D_{\alpha}$ est duale de $K_{\alpha}$.

D'après N. Steenrod [9], §34, deux champs de repères homotopes donnent lieu à deux cycles obstructeurs homologues. Une conséquence des Propriétés 2) et 3) est donc le Lemme suivant :

Lemme 3.1. - La contribution $\tilde{c}(S, X)$ ne dépend pas de la construction du repère radial sur les strates du bord $\partial S$. Autrement dit, deux champs radiaux $Z_{r}$ et $Z_{r}^{\prime}$ définis sur $S \cap \partial U$ donnent lieu à des cycles équivalents

$$
\sum_{K_{\alpha} \subset S} \mu_{\alpha} K_{\alpha}^{r-1} \sim \sum_{K_{\alpha} \subset S} \mu_{\alpha}^{\prime} K_{\alpha}^{r-1}
$$

Remarquons que, via l'isomorphisme d'Alexander $H_{2(r-1)}(S) \cong H^{2 p}(M, M \backslash$ $S)$, avec $p=m-r+1$, la classe $\tilde{c}(S, X)$ correspond à la classe notée $\hat{c}^{2 p}(S)$ dans Schwartz.

D'après le Théorème 2.3, pour montrer le Théorème 1.1 il suffit de montrer que les classes de Chern de Schwartz sont localement déterminées, ce qui est l'objet du Lemme suivant : 
Lemme 3.2. - Si $f: Y \rightarrow X$ est un morphisme propre, $S$ et $T:=f^{-1}(S)$ resp. sont éléments de décompositions admissibles de $X, Y$ resp., et $f$ se res-

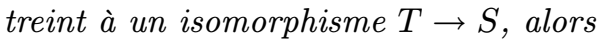

$$
f_{*} \tilde{c}(T, Y)=\tilde{c}(S, X) .
$$

Preuve. Notons $\partial S$ et $\partial T$ les bords de $S$ et $T$ respectivement, unions de strates de $X$ et $Y$ et notons $k$ la dimension (complexe) commune de $S$ et $T$. Supposons effectuée la construction des classes de Schwartz de $X$ et $Y$ par récurrence sur la dimension des strates jusqu'en dimension $k-1$ incluse. Cette construction donne lieu à deux champs de $r$-repères : l'un $Z_{r}$, défini sur un voisinage cellulaire $U$ de $\partial S$, sortant de $U$, et l'autre $W_{r}$, défini sur un voisinage cellulaire $V$ de $\partial T$, sortant de $V$. Nous avons donc la situation suivante :

Sur $S \cap \partial U$ le $r$-repère $Z_{r}$ est tangent à $S$ et entrant dans $S \backslash U$. Sur $T \cap \partial V$ le $r$-repère $W_{r}$ est tangent à $T$ et entrant dans $T \backslash V$. D'après l'hypothèse du Théorème, on a un isomorphisme des paires $(S \backslash U, S \cap \partial U) \cong(T \backslash V, T \cap \partial V)$. On conclut par le Lemme 3.1.

\section{BIBLIOGRAPHIE}

[1] P. Aluffi - «Limits of Chow groups, and a new construction of ChernSchwartz-MacPherson classes », Pure Appl. Math. Q. 2 (2006), p. 915-941.

[2] J.-P. Brasselet \& M.-H. Schwartz - «Sur les classes de Chern d'un ensemble analytique complexe », in Caractéristique d'Euler-Poincaré, Astérisque, vol. 83, Soc. Math. France, 1981, p. 93-147.

[3] W. Fulton - Intersection theory, Ergebnisse der Mathematik und ihrer Grenzgebiete (3), vol. 2, Springer, 1984.

[4] G. KenneDY - «MacPherson's Chern classes of singular algebraic varieties », Comm. Algebra 18 (1990), p. 2821-2839.

[5] R. D. MACPherson - «Chern classes for singular algebraic varieties », Ann. of Math. (2) 100 (1974), p. 423-432.

[6] M.-H. Schwartz - «Classes caractéristiques définies par une stratification d'une variété analytique complexe I », C. R. Acad. Sci. Paris 260 (1965), p. 3262-3264.

[7] _ _ "Classes caractéristiques définies par une stratification d'une variété analytique complexe II », C. R. Acad. Sci. Paris 260 (1965), p. 35353537.

[8] _ Classes de Chern des ensembles analytiques, Hermann, 2000.

[9] N. Steenrod - The Topology of Fibre Bundles, Princeton Mathematical Series, vol. 14, Princeton University Press, 1951. 